\title{
WELL-POSEDNESS OF A TWO-PHASE FLOW WITH SOLUBLE SURFACTANT
}

\author{
DIETER BOTHE, JAN PRÜSS, AND GIERI SIMONETT
}

\author{
Dedicated to Herbert Amann
}

\begin{abstract}
The presence of surfactants, ubiquitous at most fluid/liquid interfaces, has a pronounced effect on the surface tension, hence on the stress balance at the phase boundary: local variations of the capillary forces induce transport of momentum along the interface - so-called Marangoni effects. The mathematical model governing the dynamics of such systems is studied for the case in which the surfactant is soluble in one of the adjacent bulk phases. This leads to the two-phase balances of mass and momentum, complemented by a species equation for both the interface and the relevant bulk phase. Within the model, the motions of the surfactant and of the adjacent bulk fluids are coupled by means of an interfacial momentum source term that represents Marangoni stresses. Employing $L_{p}$-maximal regularity we obtain well-posedness of this model for a certain initial configuration. The proof is based on recent $L_{p}$-theory for two-phase flows without surfactant.
\end{abstract}

Mathematics Subject Classification (2000): Primary 35R35, Secondary 35Q30, 76D45, $76 \mathrm{~T} 10$.

Key words: Navier-Stokes equations, surface tension, Marangoni forces, maximal regularity, surface transport theorem

\section{Introduction.}

Surfactants are surface active agents that are adsorbed at gas/liquid or liquid/liquid interfaces, where they lower the surface tension. The presence of surfactants in multiphase systems, either in the form of unavoidable impurities, or as specific substances like emulsifiers to affect the system's behavior, has a significant influence on the shape and the dynamics of the interfaces. Indeed, resulting variations of the capillary force induce Marangoni stresses which increase the drag of fluid particles and hinder the internal circulation [7]. For example, the terminal rise velocity of bubbles or droplets can be drastically reduced, which results in larger contact times between the fluid phases and, hence, alters mass transfer.

Surfactants are soluble in at least one of the adjacent bulk phases, hence there is also exchange of surfactants between the relevant bulk phases and the interface by adsorption and desorption [8]. Along the interface surfactant is transported by convection and diffusion. Furthermore, changes of the interfacial area due to compression or stretching cause corresponding changes in the surfactant concentration.

Mathematically, the analysis of two-phases flows with surfactant corresponds to the study of free boundary problems for the Navier-Stokes equations, coupled with a diffusion equation having a dynamic condition on the free boundary.

Existence results for the one-phase Navier-Stokes equations with a free surface were obtained in $[2,5,31,32,33]$. In a series of publications, Solonnikov considered the motion of an isolated viscous incompressible drop subject to capillary forces, see [27] and the references contained therein.

There is significantly less work for the one-phase Navier-Stokes equations with Marangoni effects, which refers to situations where the surface endures tangential stresses caused by variations of surface tension. The latter can be caused by temperature gradients, for instance. This case is considered in [1], where the flow of a viscous liquid with its free surface subjected to a nonuniform thermal flux is studied; see also [19]. Assuming the Boussinesq approximation to account for density variations, stationary solutions are obtained in weighted Hölder spaces. 
The instationary situation is considered in [34], where small time existence of the free surface flow of a heat conducting viscous droplet of constant density in three dimensions is proved.

Problems involving the two-phase Navier-Stokes equations with surface tension have been investigated in $[28,29,10,9]$. In $[28,29]$ small time existence for smooth initial data as well as global existence and convergence to the equilibrium configuration for nearby initial data is proven. Small time existence in weighted Hölder spaces is obtained in [10] for incompressible fluids, while combinations of compressible and incompressible fluids are allowed in [9]. For compressible two-phase flow without surface tension but with additional energy balance see [30].

There is a large amount of literature in the Engineering Sciences on two-phase flows in the presence of surface active substances, but a rigorous mathematical analysis is still missing so far. However, interesting first numerical results in 2D are obtained in [18], while analytical calculations for bubbles in plane extensional flow of low velocity are given in [25]. In these contributions, the surfactant is considered as insoluble, a common idealization.

In the present paper we consider the two-phase Navier-Stokes equations with surfactant dependent surface tension complemented by a convection-diffusion equation inside one phase with another convection-diffusion equation on the free surface which are nonlinearly coupled. We give a derivation of the mathematical model, for which an appropriate surface transport theorem is employed. To keep the paper self-contained, a proof of the latter together with an introduction of the notion of surface divergence is included in two appendices. The main result of this paper is the existence of a unique classical solution to this free boundary problem for a certain initial configuration of the phases. The latter corresponds to situations encountered by the usual localization procedure which leads to "deformed halfspaces" for the two phases. The strategy here is to build up the proof based on recent results on two-phase flows without surfactant. This works, since, luckily, the surfactant equations are decoupled from the NavierStokes equations in the associated linear problem.

Our proof makes use of recent developments in the theory of maximal regularity for parabolic problems. For a general reference to this subject, we refer to the monograph [11]. Of particular importance for this work are the papers [12, 13, 14, 22, 23], where basic notations used throughout this paper can be found.

\section{The Mathematical Model.}

The subsequent mathematical model describes two-phase flows with a free boundary to capture the motion of a fluid particle (droplet or bubble) in an ambient fluid phase (gas or liquid). We consider isothermal flows of two immiscible Newtonian fluids with constant densities. Based on continuum mechanics, balance of mass and momentum inside the phases lead to the NavierStokes equations, i.e.

$$
\nabla \cdot u=0, \quad \partial_{t}\left(\rho_{ \pm} u\right)+\nabla \cdot\left(\rho_{ \pm} u \otimes u-S\right)=0
$$

with the stress tensor

$$
S=-\pi I+\mu_{ \pm}\left(\nabla u+(\nabla u)^{\top}\right) .
$$

These balances hold in $\Omega_{+}(t) \cup \Omega_{-}(t)$, where $\Omega_{ \pm}(t)$ are the domains occupied at time $t$ by two fluids with density $\rho_{ \pm}$and viscosity $\mu_{ \pm}$, respectively. Hence the material parameters depend on the phase. Whenever a distinction between the different phases is not necessary, the phase indices + and - are omitted. The phases are separated by a phase boundary $\Gamma(t)$ at which at least one of the material parameters has a jump-discontinuity. At this interface, the additional jump conditions

$$
\begin{aligned}
{\left[\rho\left(u-u_{\text {int }}\right)\right] n } & =0, \\
{\left[\rho u \otimes\left(u-u_{\text {int }}\right)-S\right] n } & =\sigma \kappa n+\operatorname{grad}_{\Gamma} \sigma
\end{aligned}
$$

appear, where $u_{\text {int }}$ is the interfacial velocity, $\operatorname{grad}_{\Gamma} \sigma$ denotes the surface gradient of the surface tension $\sigma$ (cf. Appendix A) and

$$
\kappa=-\operatorname{div}_{\Gamma} n
$$

is the sum of the (local) principal curvatures of the interface. More precisely, it should read $\operatorname{div}_{\Gamma(t)}$, but the dependence on $t$ is dropped whenever this is reasonable. Throughout the paper, $n$ denotes the unit normal at the interface directed into the phase $\Omega_{+}(t)$, say, in which case 
$\kappa(t, x)>0$ at $x \in \Gamma(t)$ iff $\Omega_{+}(t) \cap B_{r}(x)$ is convex for small $r>0$. The notation $[\phi]$ stands for the jump of a physical quantity $\phi$ across the interface, i.e.

$$
[\phi](t, x)=\lim _{h \rightarrow 0+}(\phi(t, x+h n)-\phi(t, x-h n)), \quad x \in \Gamma(t) .
$$

Below, it is assumed that the two-fluid system under consideration does not endure phase changes like melting or evaporation. Consequently, there are no convective fluxes across the interface, and hence the normal components of the fluid velocities are continuous at the phase boundary, and the interface itself is advected with the flow, i.e.

$$
\left(u_{+} \cdot n\right)_{\mid \Gamma(t)}=\left(u_{-} \cdot n\right)_{\mid \Gamma(t)}=V,
$$

where $V$ denotes the normal velocity of the interface. Furthermore, we assume no-slip at the interface. Then the jump conditions simplify to

$$
[u]=0, \quad[-S] n=\sigma \kappa n+\operatorname{grad}_{\Gamma} \sigma .
$$

Throughout this paper, we consider the case of a soluble surfactant, i.e. a chemical species which is adsorbed at the interface but also lives in at least one of the bulk phases. For technical simplicity, we concentrate on the case in which this species is only present in phase $\Omega_{+}$, which is also of practical relevance. The local concentration $c$ of a dilute chemical species is governed by the balance equation

$$
\partial_{t} c+\nabla \cdot(c u+J)=0
$$

where we assume that the molecular flux is given by Fick's law,

$$
J=-D \nabla c,
$$

with constant diffusivity $D>0$. This leads to the convection-diffusion equation

$$
\partial_{t} c+u \cdot \nabla c-D \Delta c=0
$$

inside $\Omega_{+}$. For more details about the continuum mechanical modeling of two-phase flows up to this point see $[16,26]$.

At the phase boundary, mass balance for a material control area, i.e. $M(t)=\Gamma(t) \cap V(t)$ with a material control volume $V(t)$, implies

$$
\frac{d}{d t} \int_{M(t)} c_{\Gamma} d \sigma_{t}=-\int_{\partial M(t)} J_{\Gamma} \cdot N d s_{t}-\int_{M(t)}[J] \cdot n d \sigma_{t} .
$$

Here $c_{\Gamma}$ denotes the surface specific concentration of the surfactant on the interface $\Gamma$ and $J_{\Gamma}$ is the interfacial molecular flux of surfactant which will be modeled by Fick's law again, i.e.

$$
J_{\Gamma}=-D_{\Gamma} \operatorname{grad}_{\Gamma} c_{\Gamma}
$$

with $D_{\Gamma}>0$. Observe also that $[J] \cdot n$ reduces to $-D(\nabla c \cdot n)_{\mid \Gamma(t)}$, understood as the $\Omega_{+}$-sided limit, here, since the species $c$ is only present in $\Omega_{+}$. Application of the surface divergence theorem (Theorem A in Appendix A), the surface transport theorem (Theorem B in Appendix B) and the usual localization procedure leads to the differential formulation of the surfactant balance. A completely Eulerian formulation is problematic since $c_{\Gamma}$ is only defined on $\operatorname{gr}(\Gamma)$. An intermediate Eulerian/Lagrangian formulation reads as

$$
\partial_{t, n} c_{\Gamma}+\operatorname{div}_{\Gamma}\left(c_{\Gamma} u_{\Gamma}\right)-c_{\Gamma} \kappa V-D_{\Gamma} \Delta_{\Gamma} c_{\Gamma}=D(\nabla c \cdot n)_{\mid \Gamma(t)},
$$

where $\partial_{t, n} c_{\Gamma}$ stands for the derivative of $c_{\Gamma}$ along a purely normal path, $u_{\Gamma}$ is the tangential part of $u$ and $\Delta_{\Gamma}$ denotes the Laplace-Beltrami operator on $\Gamma$; cf. Appendix B.

One more constitutive equation is needed to determine the surfactant distribution. Here we assume that the process of ad- and desorption of surfactant at the interface is instantaneous if viewed on the time scale of convective and diffusive transport. This leads to the additional relation

$$
c_{\Gamma}=\gamma\left(c_{\mid \Gamma(t)}\right),
$$

where it is reasonable to assume that $\gamma: \mathbb{R}_{+} \rightarrow \mathbb{R}_{+}$is $C^{2}$ with $\gamma^{\prime}>0$. In concrete constitutive equations, $\gamma$ will usually satisfy $\gamma(\infty)=c_{\Gamma}^{\infty}$, since the phase boundary has a finite capacity concerning adsorption of surfactant. This relation gets more complicated in case of liquid/liquid/surfactant-systems in which the surfactant is soluble in both phases; a survey on adsorption and partitioning of surfactants is given in [24]. 

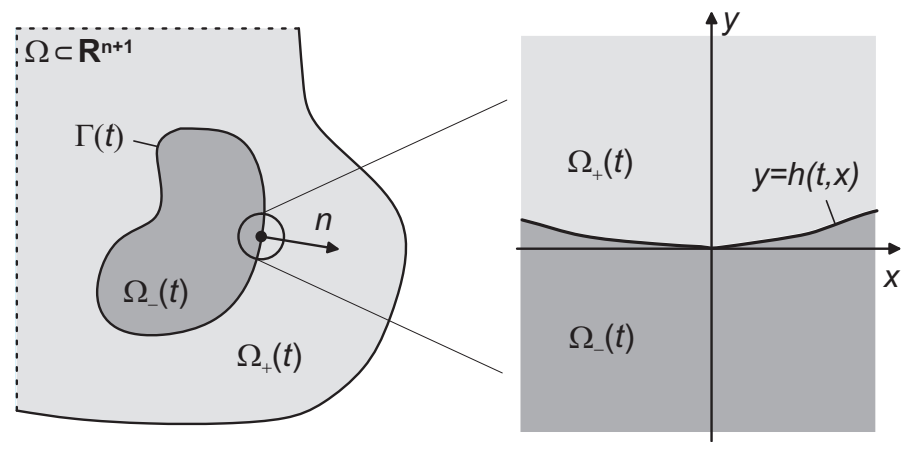

Figure 1: Configuration of the phases at the interface and localization.

Finally, the surface tension $\sigma$ is a function of $c_{\Gamma}$ and it can be significantly lowered due to the adsorption of surfactant. Below, we assume that $\sigma: \mathbb{R}_{+} \rightarrow \mathbb{R}_{+}$is $C^{2}$ with $\sigma>0$. In this situation, a nonhomogeneous surfactant distribution leads to nonzero surface gradients of the surface tension. This corresponds to so-called Marangoni forces which cause tangential stress at the phase boundary and can trigger currents along the interface, the so-called Marangoni convection. This back-effect of the surfactant distribution on the hydrodynamics is mediated via the surface gradient of $\sigma$ within the momentum jump condition. The full model of a twophase flow with soluble surfactant now reads as

\section{Balance equations.}

$$
\begin{aligned}
\nabla \cdot u=0 & \text { for } \quad t>0, x \in \Omega_{ \pm}(t) \\
\partial_{t}\left(\rho_{ \pm} u\right)+\nabla \cdot\left(\rho_{ \pm} u \otimes u-S\right)=0 & \text { for } \quad t>0, x \in \Omega_{ \pm}(t) \\
\partial_{t} c+u \cdot \nabla c-D \Delta c=0 & \text { for } \quad t>0, x \in \Omega_{+}(t) .
\end{aligned}
$$

Free boundary conditions.

$$
\begin{array}{r}
{[u]=0, \quad V=u \cdot n \quad \text { for } \quad t>0, x \in \Gamma(t),} \\
{[-S] n=\sigma\left(c_{\Gamma}\right) \kappa n+\sigma^{\prime}\left(c_{\Gamma}\right) \operatorname{grad}_{\Gamma} c_{\Gamma} \quad \text { for } \quad t>0, x \in \Gamma(t),} \\
D(\nabla c \cdot n)_{\mid \Gamma(t)}=\partial_{t, n} c_{\Gamma}+\operatorname{div}_{\Gamma}\left(c_{\Gamma} u_{\Gamma}\right)-c_{\Gamma} \kappa V-D_{\Gamma} \Delta_{\Gamma} c_{\Gamma} \quad \text { for } \quad t>0, x \in \Gamma(t), \\
c_{\Gamma}(t, x)=\gamma(c(t, x)) \quad \text { for } \quad t>0, x \in \Gamma(t) .
\end{array}
$$

\section{Initial conditions.}

$$
\begin{aligned}
u(0, x) & =u_{0}(x), \quad \text { for } \quad x \in \Omega_{ \pm}(0), \\
c(0, x) & =c_{0}(x) \quad \text { for } \quad x \in \Omega_{+}(0), \\
\Gamma(0)=\Gamma_{0}, \quad c_{\Gamma}(0, x) & =c_{\Gamma}^{0}(x) \quad \text { for } \quad x \in \Gamma_{0} .
\end{aligned}
$$

Recall that $S$ is given by

$$
S=-\pi I+S^{0} \quad \text { with } \quad S^{0}=\mu_{ \pm}\left(\nabla u+(\nabla u)^{\top}\right) .
$$

\section{Main Result: Small Time Existence in a Deformed Halfspace.}

The principal aim of this investigation is to obtain local existence of classical solutions for $\Gamma_{0}$ being a closed manifold such that the connected component of $\mathbb{R}^{n+1} \backslash \Gamma_{0}$ represents a fluid particle like a drop or a bubble. This general situation can be treated by a localization procedure, which will be carried out in a forthcoming paper. Here, we are interested in the 
model problem which occurs by localization at points on $\Gamma_{0}$; cf. figure 1 . In this case, $\Omega_{ \pm}$are deformed halfspaces and $\Gamma_{0}$ is close to a plane, say $\mathbb{R}^{n}$, i.e. $\Gamma_{0}$ is a graph over $\mathbb{R}^{n}$ given by a function $h_{0}$ with $\left|\nabla h_{0}\right|_{\infty}$ small. The main result for this model problem reads as follows.

Theorem 1. Let $\rho_{ \pm}, \mu_{ \pm}, D, D_{\Gamma}>0$ and $\gamma, \sigma \in C^{2}\left(\mathbb{R}_{+}\right)$with $\sigma>0$. Fix $p>n+3$ and suppose the following conditions to hold.

Regularity of initial data:

$$
\begin{aligned}
& h_{0} \in W_{p}^{3-2 / p}\left(\mathbb{R}^{n}\right), \quad \Gamma_{0}=\operatorname{gr}\left(h_{0}\right), u_{0} \in W_{p}^{2-2 / p}\left(\mathbb{R}^{n+1} \backslash \Gamma_{0} ; \mathbb{R}^{n+1}\right), \\
& c_{0}-\bar{c} \in W_{p}^{2-2 / p}\left(\Omega_{+}^{0}\right), \quad c_{\Gamma}^{0}-\bar{c}_{\Gamma} \in W_{p}^{2-2 / p}\left(\Gamma_{0}\right)
\end{aligned}
$$

with appropriate $\bar{c}, \bar{c}_{\Gamma} \in \mathbb{R}$ and $\Omega_{+}^{0}=\left\{(x, y) \in \mathbb{R}^{n+1}: x \in \mathbb{R}^{n}, y>h_{0}(x)\right\}$.

Compatibility condition:

$$
\begin{gathered}
\nabla \cdot u_{0}=0, \quad\left[S_{\mid t=0}^{0} n-\left\langle n, S_{\mid t=0}^{0} n\right\rangle n\right]=-\sigma^{\prime}\left(c_{\Gamma}^{0}\right) \operatorname{grad}_{\Gamma_{0}} c_{\Gamma}^{0}, \\
{\left[u_{0}\right]=0, \quad c_{\Gamma}^{0}=\gamma\left(c_{0 \mid \Gamma_{0}}\right), \quad \bar{c}_{\Gamma}=\gamma(\bar{c}) .}
\end{gathered}
$$

Then there is $\eta>0$ such that the following holds. Given $h_{0}$ with $\left|\nabla h_{0}\right| \leq \eta$ and $c_{\Gamma}^{0} \geq 0$ with

$$
\sup _{x \in \Gamma_{0}} c_{\Gamma}^{0}-\inf _{x \in \Gamma_{0}} c_{\Gamma}^{0} \leq \eta,
$$

there exist $t_{0}=t_{0}\left(u_{0}, h_{0}, c_{0}, c_{\Gamma}^{0}\right)$ and a classical solution $\left(u, \pi, c, c_{\Gamma}\right)$ of $(1)-(4)$ in $\left(0, t_{0}\right)$. The solution is unique within the class of maximal regularity as described in Theorem 2 below.

The proof of Theorem 1 consists of three main steps as given in the subsections to follow.

\subsection{Reduction to a Halfspace.}

Suppose that $\Gamma(t)$ is a graph over $\mathbb{R}^{n}$, parameterized as

$$
\Gamma(t)=\left\{(x, h(t, x)): x \in \mathbb{R}^{n}\right\} \quad \text { for } t \in J=[0, T]
$$

with $\Omega_{+}(t)$ lying "above" $\Gamma(t)$, i.e.

$$
\Omega_{ \pm}(t)=\left\{(x, y) \in \mathbb{R}^{n} \times \mathbb{R}: y \gtrless h(t, x)\right\} \quad \text { for } t \in J .
$$

Reduction from deformed into true halfspaces is achieved by means of the transformations

$$
\begin{aligned}
v(t, x, y) & =\left[\begin{array}{c}
u_{1}(t, x, h(t, x)+y) \\
\vdots \\
u_{n}(t, x, h(t, x)+y)
\end{array}\right] \\
w(t, x, y) & =u_{n+1}(t, x, h(t, x)+y) \\
p(t, x, y) & =\pi(t, x, h(t, x)+y)
\end{aligned}
$$

where $t \in J, x \in \mathbb{R}^{n}, y \in \mathbb{R}$ and

$$
\begin{aligned}
\tilde{c}(t, x, y) & =c(t, x, h(t, x)+y)-\bar{c} \\
\tilde{c}_{\Gamma}(t, x) & =c_{\Gamma}(t, x, h(t, x))-\gamma(\bar{c}),
\end{aligned}
$$

where $t \in J, x \in \mathbb{R}^{n}, y \in \mathbb{R}_{+}=[0, \infty)$. Notice that we passed to concentration differences w.r. to the "values at infinity" and that now $(x, y)$ instead of $x$ denotes a point in $\mathbb{R}^{n+1}$. For the two-phase balance equations for mass and momentum, this conversion has already been carried out in [14]. Therefore, we concentrate on those calculations needed to transform the surfactant balance. Since the orientation of $\Gamma(t)$ is such that the normal field is exterior to $\Omega_{-}(t)$, the unit normal at the point $(x, h(t, x))$ is given by

$$
n(t, x)=\beta(t, x)^{-1 / 2}\left[\begin{array}{c}
-\nabla_{x} h(t, x) \\
1
\end{array}\right] \quad \text { with } \beta(t, x)=1+\left|\nabla_{x} h(t, x)\right|^{2} .
$$

The normal velocity $V$ of $\Gamma(\cdot)$ is

$$
V(t, x)=\beta(t, x)^{-1 / 2} \partial_{t} h(t, x) .
$$


Together with the kinematic condition $V=(u \cdot n)_{\mid \Gamma}$ this yields the transport equation

$$
\partial_{t} h+v_{\mid y=0} \cdot \nabla_{x} h=w_{\mid y=0},
$$

which describes the evolution of $h$. Note that $u$ is contiuous across $\Gamma(t)$, hence $v_{\mid y=0}$ and $w_{\mid y=0}$ are well-defined.

According to (24) in Appendix A, the curvature of $\Gamma(t)$ at $x \in \Gamma(t)$ can be calculated as

$$
\kappa(t, x)=-\operatorname{div}_{\Gamma(t)} n(t, x)=\nabla_{x} \cdot\left(\beta(t, x)^{-1 / 2} \nabla_{x} h(t, x)\right),
$$

hence

$$
\kappa=\Delta_{x} h-G_{\kappa}(h)
$$

with

$$
G_{\kappa}(h)=\left(1-\beta^{-1 / 2}\right) \Delta_{x} h+\beta^{-3 / 2}\left\langle\nabla_{x} h, \nabla_{x}^{2} h \nabla_{x} h\right\rangle .
$$

Differentiation of $c(t, x, y)=\tilde{c}(t, x, y-h(t, x))+\bar{c}$ yields

$$
u \cdot \nabla c=v \cdot \nabla_{x} \tilde{c}+\left(w-v \cdot \nabla_{x} h\right) \partial_{y} \tilde{c}
$$

as well as

$$
\Delta c=\Delta_{x} \tilde{c}+\beta \partial_{y}^{2} \tilde{c}-2\left\langle\nabla_{x} \partial_{y} \tilde{c}, \nabla_{x} h\right\rangle-\partial_{y} \tilde{c} \Delta_{x} h,
$$

where the corresponding arguments have to be used. Therefore, the surfactant balance in $\Omega_{+}(t)$ transforms into the quasilinear partial differential equation

$$
\partial_{t} \tilde{c}-D \Delta_{x} \tilde{c}-D \partial_{y}^{2} \tilde{c}=F_{c}(v, \tilde{c}, h), \quad t \in J, x \in \mathbb{R}^{n}, y \in \mathbb{R} .
$$

Here, the nonlinearity is given by

$$
F_{c}(v, \tilde{c}, h)=-v \cdot \nabla_{x} \tilde{c}+D\left((\beta-1) \partial_{y}^{2} \tilde{c}-2\left\langle\nabla_{x} \partial_{y} \tilde{c}, \nabla_{x} h\right\rangle-\partial_{y} \tilde{c} \Delta_{x} h\right) .
$$

For the transformation of the surfactant balance on $\Gamma(t)$, we prefer to use the formulation

$$
\frac{D c_{\Gamma}}{D t}+c_{\Gamma} \operatorname{div}_{\Gamma} u_{\Gamma}-c_{\Gamma} \kappa V-D_{\Gamma} \Delta_{\Gamma} c_{\Gamma}=D(\nabla c \cdot n)_{\mid \Gamma},
$$

i.e. the one which employs the full Lagrangian derivative

$$
\frac{D c_{\Gamma}}{D t}(t, x, h(t, x))=\frac{d}{d s} c_{\Gamma}(t+s, \xi(t+s), \eta(t+s))_{\mid s=0}
$$

with $(\xi, \eta)$ the solution of

$$
\begin{array}{ll}
\dot{\xi}_{k}(s)=u_{k}(s, \xi(s), \eta(s)), & \xi_{k}(t)=x_{k} \text { for } k=1, \ldots, n, \\
\dot{\eta}(s)=u_{n+1}(s, \xi(s), \eta(s)), & \eta(t)=h(t, x) .
\end{array}
$$

Exploiting (5) it follows that $\eta(s)=h(s, \xi(s))$, hence

$$
\begin{aligned}
\frac{D c_{\Gamma}}{D t}(t, x, h(t, x)) & =\frac{d}{d s} c_{\Gamma}(t+s, \xi(t+s), h(t+s, \xi(t+s)))_{\mid s=0} \\
& =\frac{d}{d s} \tilde{c}_{\Gamma}(t+s, \xi(t+s))_{\mid s=0} \\
& =\partial_{t} \tilde{c}_{\Gamma}(t, x)+v(t, x) \cdot \nabla_{x} \tilde{c}_{\Gamma}(t, x) .
\end{aligned}
$$

We will now compute $\operatorname{div}_{\Gamma} u=\operatorname{div}_{\Gamma} u_{\Gamma}-\kappa V$ in the new variables. According to (19), the surface divergence $\operatorname{div}_{\Gamma} u$ of the vector field $u$ is given by

$$
\begin{aligned}
\operatorname{div}_{\Gamma} u(x, h(x)) & =\sum_{i, j=1}^{n} g^{i j}(x)\left\langle\partial_{i} u(x, h(x)),\left(e_{j}, \partial_{j} h(x)\right)\right\rangle \\
& =\sum_{i, j=1}^{n}\left(\delta_{i j}-\beta^{-1} \partial_{i} h(x) \partial_{j} h(x)\right)\left(\partial_{i} v_{j}(x, 0)+\partial_{i} w(x, 0) \partial_{j} h(x)\right) \\
& =\nabla_{x} \cdot v(x, 0)-\beta^{-1}\left\langle\nabla_{x} w(x, 0)-\nabla_{x} v(x, 0) \nabla_{x} h(x), \nabla_{x} h(x)\right\rangle .
\end{aligned}
$$

In the computations above we have suppressed the variable $t$.

The Laplace-Beltrami operator (see Appendix A) transforms as

$$
\Delta_{\Gamma} c_{\Gamma}=\beta^{-1 / 2} \nabla_{x} \cdot\left(\beta^{1 / 2} \nabla_{x} \tilde{c}_{\Gamma}-\beta^{-1 / 2}\left\langle\nabla_{x} h, \nabla_{x} \tilde{c}_{\Gamma}\right\rangle \nabla_{x} h\right) .
$$


A direct calculation shows that

$$
\Delta_{\Gamma} c_{\Gamma}=\Delta_{x} \tilde{c}_{\Gamma}-\beta^{-1}\left\langle\nabla_{x} h, \nabla_{x}^{2} \tilde{c}_{\Gamma} \nabla_{x} h+\Delta_{x} h \nabla_{x} \tilde{c}_{\Gamma}\right\rangle+\beta^{-2}\left\langle\nabla_{x}^{2} h \nabla_{x} h, \nabla_{x} h\right\rangle \nabla_{x} h \cdot \nabla_{x} \tilde{c}_{\Gamma} .
$$

Here, $\nabla_{x}^{2} \tilde{c}_{\Gamma}$ and $\nabla_{x}^{2} h$ denote the matrix of all (mixed) second-order derivatives of $\tilde{c}_{\Gamma}$ and $h$, respectively. Finally, exploitation of

$$
\nabla c(t, x, y)=\left(\nabla_{x} \tilde{c}-\partial_{y} \tilde{c} \nabla_{x} h, \partial_{y} \tilde{c}\right)(t, x, y-h(t, x))
$$

yields

$$
(\nabla c \cdot n)_{\mid \Gamma}=\left(\beta^{1 / 2} \partial_{y} \tilde{c}-\beta^{-1 / 2} \nabla_{x} \tilde{c} \cdot \nabla_{x} h\right)_{\mid y=0} \cdot
$$

Altogether, this leads to the quasilinear partial differential equation

$$
\partial_{t} \tilde{c}_{\Gamma}-D_{\Gamma} \Delta_{x} \tilde{c}_{\Gamma}=G_{c}\left(v, w, \tilde{c}_{\Gamma}, \tilde{c}, h\right), \quad t \in J, x \in \mathbb{R}^{n}
$$

with

$$
\begin{aligned}
G_{c}\left(v, w, \tilde{c}_{\Gamma}, \tilde{c}, h\right)= & -v \cdot \nabla_{x} \tilde{c}_{\Gamma}-\left(\tilde{c}_{\Gamma}+\bar{c}_{\Gamma}\right)\left(\nabla_{x} \cdot v+\beta^{-1}\left\langle\nabla_{x} w-\nabla_{x} v \nabla_{x} h, \nabla_{x} h\right\rangle\right) \\
& -D_{\Gamma} \beta^{-1}\left\langle\nabla_{x} h, \nabla_{x}^{2} \tilde{c}_{\Gamma} \nabla_{x} h+\Delta_{x} h \nabla_{x} \tilde{c}_{\Gamma}\right\rangle \\
& +D_{\Gamma} \beta^{-2}\left\langle\nabla_{x}^{2} h \nabla_{x} h, \nabla_{x} h\right\rangle \nabla_{x} h \cdot \nabla_{x} \tilde{c}_{\Gamma} \\
& +D \beta^{1 / 2} \partial_{y} \tilde{c}_{\mid y=0}-D \beta^{-1 / 2} \nabla_{x} \tilde{c}_{\mid y=0} \cdot \nabla_{x} h .
\end{aligned}
$$

The nonlinear boundary condition for the transformed concentration reads as

$$
\tilde{c}_{\Gamma}=\gamma\left(\bar{c}+\tilde{c}_{\mid y=0}\right)-\gamma(\bar{c}) .
$$

To obtain the linearized version, this is rewritten as

$$
\tilde{c}_{\Gamma}-\gamma_{0} \tilde{c}_{\mid y=0}=G_{\gamma}\left(\tilde{c}_{\mid y=0}\right)
$$

with $\gamma_{0}=\gamma^{\prime}(\bar{c})$ and

$$
G_{\gamma}\left(\tilde{c}_{\mid y=0}\right)=\gamma\left(\bar{c}+\tilde{c}_{\mid y=0}\right)-\gamma(\bar{c})-\gamma^{\prime}(\bar{c})\left(\tilde{c}_{\mid y=0}\right) .
$$

To exploit the results from [14] for constant surface tension, those terms have to be specified that correspond to the changes in the momentum jump condition in case $\sigma$ depends on the surfactant concentration $c_{\Gamma}$. For this purpose, let $\sigma_{0}=\sigma\left(\bar{c}_{\Gamma}\right)$ and rewrite the second equation in $(2)$ as

$$
[-S] n-\sigma_{0} \kappa n=\left(\sigma\left(c_{\Gamma}\right)-\sigma_{0}\right) \kappa n+\sigma^{\prime}\left(c_{\Gamma}\right) \operatorname{grad}_{\Gamma} c_{\Gamma}
$$

The right-hand side consists of a tangential part corresponding to $v$ and a normal part corresponding to $w$. After application of the above transformations, this leads to nonlinearities $G_{v}^{\text {surf }}$ and $G_{w}^{\text {surf }}$ which are given by

$$
\begin{aligned}
G_{v}^{\text {surf }}\left(\tilde{c}_{\Gamma}, h\right)=( & \left.\sigma\left(\tilde{c}_{\Gamma}\right)-\sigma_{0}\right)\left(G_{\kappa}(h)-\Delta_{x} h\right) \nabla_{x} h \\
& +\sigma^{\prime}\left(\tilde{c}_{\Gamma}\right) \nabla_{x} \tilde{c}_{\Gamma}-\beta^{-1} \sigma^{\prime}\left(\tilde{c}_{\Gamma}\right)\left(\nabla_{x} \tilde{c}_{\Gamma} \cdot \nabla_{x} h\right) \nabla_{x} h, \\
G_{w}^{\mathrm{surf}}\left(\tilde{c}_{\Gamma}, h\right)=( & \left.\sigma\left(\tilde{c}_{\Gamma}\right)-\sigma_{0}\right)\left(\Delta_{x} h-G_{\kappa}(h)\right)+\beta^{-1} \sigma^{\prime}\left(\tilde{c}_{\Gamma}\right) \nabla_{x} \tilde{c}_{\Gamma} \cdot \nabla_{x} h .
\end{aligned}
$$

Before writing down the full system of transformed equations, we will introduce some more notation. We set

$$
\begin{aligned}
& \mathbb{R}^{n} \times \dot{\mathbb{R}}:=\left\{(x, y) \in \mathbb{R}^{n} \times \mathbb{R}: x \in \mathbb{R}^{n}, y \neq 0\right\}, \\
& \mathbb{H}_{+}^{n+1}:=\mathbb{R}^{n} \times(0, \infty), \\
& \mathbb{H}_{-}^{n+1}:=\mathbb{R}^{n} \times(-\infty, 0) .
\end{aligned}
$$

Suppose that $f: \mathbb{R}^{n} \times \dot{\mathbb{R}} \rightarrow \mathbb{R}^{k}$ is some given function. Then

$$
f_{+}:=\left.f\right|_{\mathbb{H}_{+}^{n+1}}, \quad f_{-}:=\left.f\right|_{\mathbb{H}_{-}^{n+1}}
$$

denote the restrictions of $f$ to $\mathbb{H}_{+}^{n+1}$ and $\mathbb{H}_{-}^{n+1}$, respectively. It is clear that $f$ completely determines the functions $f_{+}$and $f_{-}$, and vice versa. Moreover, the mapping

$$
f \mapsto\left(f_{+}, f_{-}\right), \quad W_{p}^{s}\left(\mathbb{R}^{n} \times \dot{\mathbb{R}} ; \mathbb{R}^{k}\right) \rightarrow W_{p}^{s}\left(\mathbb{H}_{+}^{n+1} ; \mathbb{R}^{k}\right) \times W_{p}^{s}\left(\mathbb{H}_{-}^{n+1} ; \mathbb{R}^{k}\right)
$$


defines an isometric isomorphism, where $W_{p}^{s}$ are the usual Sobolev-Slobodeskii spaces.

Next suppose that we are given a pair of positive numbers, say $\mu=\left(\mu_{+}, \mu_{-}\right)$, and a function $f: \mathbb{R}^{n} \times \dot{\mathbb{R}} \rightarrow \mathbb{R}^{k}$. Then

$$
\mu f(x, y):= \begin{cases}\mu_{+} f_{+}(x, y) & \text { if } y>0, \\ \mu_{-} f_{-}(x, y) & \text { if } y<0 .\end{cases}
$$

If the function $f$ has one-sided limits at $y=0$ these are denoted by $f_{+}(x, 0)$ and $f_{-}(x, 0)$, respectively. Then we set

$$
[\mu f](x):=\mu_{+} f_{+}(x, 0)-\mu_{-} f_{-}(x, 0), \quad x \in \mathbb{R}^{n} .
$$

If $[f]=0$ we write

$$
f(x):=f(x, 0):=f_{+}(x, 0)=f_{-}(x, 0) .
$$

Note that in this case we have $[\mu f]=[\mu] f$ for any pair $\mu=\left(\mu_{+}, \mu_{-}\right) \in \mathbb{R}^{2}$.

Combining the transformed equations derived above with those from [14], we end up with the following coupled system of nonlinear equations.

\section{Differential Equations.}

$$
\begin{aligned}
\rho \partial_{t} v-\mu \Delta v+\nabla_{x} p & =F_{v}(v, w, p, h) & & \text { in } J \times \mathbb{R}^{n} \times \dot{\mathbb{R}} \\
\rho \partial_{t} w-\mu \Delta w+\partial_{y} p & =F_{w}(v, w, h) & & \text { in } J \times \mathbb{R}^{n} \times \dot{\mathbb{R}} \\
\nabla_{x} \cdot p+\partial_{y} p & =F_{p}(v, h) & & \text { in } J \times \mathbb{R}^{n} \times \dot{\mathbb{R}} \\
\partial_{t} \tilde{c}-D \Delta \tilde{c} & =F_{c}(v, \tilde{c}, h) & & \text { in } J \times \mathbb{H}_{+}^{n+1} \\
\partial_{t} h-w & =G_{h}(v, h) & & \text { on } J \times \mathbb{R}^{n} \\
\partial_{t} \tilde{c}_{\Gamma}-D_{\Gamma} \Delta_{x} \tilde{c}_{\Gamma} & =G_{c}\left(v, w, \tilde{c}_{\Gamma}, \tilde{c}, h\right) & & \text { on } J \times \mathbb{R}^{n} \\
-\left[\mu \partial_{y} v\right]-[\mu] \nabla_{x} w & =G_{v}(v, w,[p], h)+G_{v}^{\text {surf }}\left(\tilde{c}_{\Gamma}, h\right) & & \text { on } J \times \mathbb{R}^{n} \\
-2\left[\mu \partial_{y} w\right]+[p]-\sigma_{0} \Delta h & =G_{w}(v, w, h)+G_{v}^{\operatorname{surf}}\left(\tilde{c}_{\Gamma}, h\right) & & \text { on } J \times \mathbb{R}^{n}
\end{aligned}
$$

\section{Boundary conditions.}

$$
[v]=0, \quad[w]=0, \quad \tilde{c}_{\Gamma}-\gamma_{0} \tilde{c}_{\mid y=0}=G_{\gamma}\left(\tilde{c}_{\mid y=0}\right) \quad \text { on } \quad J \times \mathbb{R}^{n} .
$$

Here $G_{\kappa}, F_{c}, G_{c}$ and $G_{\gamma}$ are given by (7), (8), (9) and (10), respectively. The other nonlinearities have been computed in [14] and are given by

$$
\begin{aligned}
F_{v}(v, w, p, h)=\mu & \left(-2\left(\nabla_{x} h \cdot \nabla_{x}\right) \partial_{y} v+\left|\nabla_{x} h\right|^{2} \partial_{y}^{2} v-\Delta_{x} h \partial_{y} v\right)+\nabla_{x} h \partial_{y} p \\
& +\rho\left(-\left(v \cdot \nabla_{x}\right) v+\left(v \cdot \nabla_{x} h\right) \partial_{y} v-w \partial_{y} v+\left(w-v \cdot \nabla_{x} h\right) \partial_{y} v\right), \\
F_{w}(v, w, p, h)=\mu & \left(-2\left(\nabla_{x} h \cdot \nabla_{x}\right) \partial_{y} w+\left|\nabla_{x} h\right|^{2} \partial_{y}^{2} w-\Delta_{x} h \partial_{y} w\right) \\
& +\rho\left(-\left(v \cdot \nabla_{x}\right) w+\left(v \cdot \nabla_{x} h\right) \partial_{y} w-w \partial_{y} w+\left(w-v \cdot \nabla_{x} h\right) \partial_{y} w\right), \\
F_{p}(v, h)= & \nabla_{x} h \cdot \partial_{y} v \\
G_{h}(v, h)=- & -\nabla_{x} h \cdot v, \\
G_{v}(v, w,[p], h)= & \sigma_{0}\left(G_{\kappa}(h)-\Delta_{x} h\right) \nabla_{x} h-\left[\mu \partial_{y} w\right] \nabla_{x} h+[p] \nabla_{x} h \\
& -[\mu]\left(\nabla_{x} v+\left(\nabla_{x} v\right)^{\top}\right) \nabla_{x} h+\left|\nabla_{x} h\right|^{2}\left[\mu \partial_{y} v\right]+\nabla_{x} h \cdot\left[\mu \partial_{y} v\right] \nabla_{x} h, \\
G_{w}(v, w, h)= & -\sigma_{0} G_{\kappa}(h)-[\mu] \nabla_{x} h \cdot \nabla_{x} w+\left|\nabla_{x} h\right|^{2}\left[\mu \partial_{y} w\right]-\nabla_{x} h \cdot\left[\mu \partial_{y} v\right] .
\end{aligned}
$$

\subsection{The associated linear problem.}

According to Section 3.1, the linear system associated with (11), (12) has the following form. 
Differential Equations.

$$
\begin{aligned}
& \rho \partial_{t} v-\mu \Delta v+\nabla_{x} p=f_{v} \quad \text { in } J \times \mathbb{R}^{n} \times \dot{\mathbb{R}} \\
& \rho \partial_{t} w-\mu \Delta w+\partial_{y} p=f_{w} \quad \text { in } J \times \mathbb{R}^{n} \times \dot{\mathbb{R}} \\
& \nabla_{x} \cdot v+\partial_{y} w=f_{p} \quad \text { in } \quad J \times \mathbb{R}^{n} \times \dot{\mathbb{R}} \\
& \partial_{t} \tilde{c}-D \Delta \tilde{c}=f_{c} \quad \text { in } \quad J \times \mathbb{H}_{+}^{n+1} \\
& \partial_{t} h-w=g_{h} \quad \text { on } \quad J \times \mathbb{R}^{n} \\
& \partial_{t} \tilde{c}_{\Gamma}-D_{\Gamma} \Delta_{x} \tilde{c}_{\Gamma}=g_{c} \quad \text { on } J \times \mathbb{R}^{n} \\
& -\left[\mu \partial_{y} v\right]-[\mu] \nabla_{x} w=g_{v} \quad \text { on } J \times \mathbb{R}^{n} \\
& -2\left[\mu \partial_{y} w\right]+[p]-\sigma_{0} \Delta h=g_{w} \quad \text { on } J \times \mathbb{R}^{n}
\end{aligned}
$$

\section{Boundary Conditions.}

$$
[v]=0, \quad[w]=0, \quad \tilde{c}_{\Gamma}-\gamma_{0} \tilde{c}=g_{\gamma} \quad \text { on } \quad J \times \mathbb{R}^{n} .
$$

\section{Initial Conditions.}

$$
\begin{array}{rlrl}
v_{\mid t=0}=v_{0}, & w_{\mid t=0}=w_{0} & \text { on } \quad \mathbb{R}^{n} \times \dot{\mathbb{R}}, \\
\tilde{c}_{\mid t=0} & =\tilde{c}_{0} & & \text { on } \quad \mathbb{H}_{+}^{n+1}, \\
h_{\mid t=0}=h_{0}, & \tilde{c}_{\Gamma \mid t=0}=\tilde{c}_{\Gamma}^{0} & & \text { on } \quad \mathbb{R}^{n} .
\end{array}
$$

Maximal regularity in an appropriate $L_{p}$-setting for this problem is given by the following result. Before stating this result we introduce some function spaces. Let $\dot{H}_{p}^{1}\left(\mathbb{H}_{ \pm}^{n+1}\right)$ denote the closure of $C_{c}^{\infty}\left(\overline{\mathbb{H}_{ \pm}^{n+1}}\right)$ w.r. to the $L_{p^{-}}$norm of the gradient and let ${ }_{0} \dot{H}_{p}^{1}\left(\mathbb{H}_{ \pm}^{n+1}\right)$ be the closure of $C_{c}^{\infty}\left(\mathbb{H}_{ \pm}^{n+1}\right)$ w.r. to the same norm. Moreover, let $\dot{H}_{p}^{-1}\left(\mathbb{H}_{ \pm}^{n+1}\right)$ be the dual of ${ }_{0} \dot{H}_{p}^{1}\left(\mathbb{H}_{ \pm}^{n+1}\right)$.

Theorem 2. Let $\rho_{ \pm}, \mu_{ \pm}, D, D_{\Gamma}, \gamma_{0}, \sigma_{0}>0$. Let $1<p<\infty$ with $p \neq 3 / 2,3$ and $J=[0, T]$. Then problem (13)-(15) admits a unique solution $\left(v, w, p, h, \tilde{c}, \tilde{c}_{\Gamma}\right)$ such that

$$
\begin{aligned}
& (v, w) \in H_{p}^{1}\left(J ; L_{p}\left(\mathbb{R}^{n} \times \dot{\mathbb{R}} ; \mathbb{R}^{n+1}\right)\right) \cap L_{p}\left(J ; H_{p}^{2}\left(\mathbb{R}^{n} \times \dot{\mathbb{R}} ; \mathbb{R}^{n+1}\right)\right), \\
& p_{ \pm} \in L_{p}\left(J ; \dot{H}_{p}^{1}\left(\mathbb{H}_{ \pm}^{n+1} ; \mathbb{R}\right)\right), \\
& {[p] \in W_{p}^{1 / 2-1 / 2 p}\left(J ; L_{p}\left(\mathbb{R}^{n}\right)\right) \cap L_{p}\left(J ; W_{p}^{1-1 / p}\left(\mathbb{R}^{n}\right)\right),} \\
& h \in W_{p}^{2-1 / 2 p}\left(J ; L_{p}\left(\mathbb{R}^{n}\right)\right) \cap H_{p}^{1}\left(J ; W_{p}^{2-1 / p}\left(\mathbb{R}^{n}\right)\right) \cap L_{p}\left(J ; W_{p}^{3-1 / p}\left(\mathbb{R}^{n}\right)\right), \\
& \tilde{c} \in H_{p}^{1}\left(J ; L_{p}\left(\mathbb{H}_{+}^{n+1}\right)\right) \cap L_{p}\left(J ; H_{p}^{2}\left(\mathbb{H}_{+}^{n+1}\right)\right), \\
& \tilde{c}_{\Gamma} \in H_{p}^{1}\left(J ; L_{p}\left(\mathbb{R}^{n}\right)\right) \cap L_{p}\left(J ; H_{p}^{2}\left(\mathbb{R}^{n}\right)\right)
\end{aligned}
$$

if and only if the data of the problem satisfy

a) $\left(f_{v}, f_{w}\right) \in L_{p}\left(J ; L_{p}\left(\mathbb{R}^{n} \times \dot{\mathbb{R}} ; \mathbb{R}^{n+1}\right)\right)$,

b) $\left(v_{0}, w_{0}\right) \in W_{p}^{2-2 / p}\left(\mathbb{R}^{n} \times \dot{\mathbb{R}} ; \mathbb{R}^{n+1}\right), h_{0} \in W_{p}^{3-2 / p}\left(\mathbb{R}^{n}\right)$,

$$
\tilde{c}_{0} \in W_{p}^{2-2 / p}\left(\mathbb{H}_{+}^{n+1}\right), \tilde{c}_{\Gamma}^{0} \in W_{p}^{2-2 / p}\left(\mathbb{R}^{n}\right),
$$

c) $\left(f_{p}\right)^{ \pm} \in H_{p}^{1}\left(J ; \dot{H}_{p}^{-1}\left(\mathbb{H}_{ \pm}^{n+1}\right)\right) \cap L_{p}\left(J ; H_{p}^{1}\left(\mathbb{H}_{ \pm}^{n+1}\right)\right)$,

d) $f_{c} \in L_{p}\left(J ; L_{p}\left(\mathbb{H}_{+}^{n+1}\right)\right)$,

e) $\left(g_{v}, g_{w}\right) \in W_{p}^{1 / 2-1 / 2 p}\left(J ; L_{p}\left(\mathbb{R}^{n} ; \mathbb{R}^{n+1}\right)\right) \cap L_{p}\left(J ; W_{p}^{1-1 / p}\left(\mathbb{R}^{n} ; \mathbb{R}^{n+1}\right)\right)$, 
f) $g_{h} \in W_{p}^{1-1 / 2 p}\left(J ; L_{p}\left(\mathbb{R}^{n}\right)\right) \cap L_{p}\left(J ; W_{p}^{2-1 / p}\left(\mathbb{R}^{n}\right)\right)$,

g) $g_{c} \in L_{p}\left(J ; L_{p}\left(\mathbb{R}^{n}\right)\right)$,

h) $g_{\gamma} \in W_{p}^{1-1 / 2 p}\left(J ; L_{p}\left(\mathbb{R}^{n}\right)\right) \cap L_{p}\left(J ; W_{p}^{2-1 / p}\left(\mathbb{R}^{n}\right)\right)$,

and the compatibility conditions

i) $\left[v_{0}\right]=0,\left[w_{0}\right]=0$ if $p>3 / 2$,

j) $\tilde{c}_{0 \mid y=0}-\tilde{c}_{\Gamma}^{0}=g_{\gamma \mid t=0}$ if $p>3 / 2$,

k) $\nabla_{x} \cdot v_{0}^{ \pm}+\partial_{y} w_{0}^{ \pm}=f_{p \mid t=0}^{ \pm}$in $\dot{H}_{p}^{-1}\left(\mathbb{H}_{ \pm}^{n+1}\right)$

l) $-\left[\mu \partial_{y} v_{0}\right]-[\mu] \nabla_{x} w_{0}=g_{v \mid t=0}$ if $p>3$.

Proof. Concerning the proof, notice first that the "only if"-part follows by taking traces; see section 5 in [12]. For the "if"-part observe that problem (13)-(15) consists of two decoupled subsystems. The first one involves only $(u, p, h)$ and the corresponding result is Theorem 3.3 in [14]. The second one is built by the surfactant equations, i.e. those for $\tilde{c}$ and $\tilde{c}_{\Gamma}$. Here the desired maximal regularity follows from that of the diffusion equation on $\mathbb{R}^{n}$ and on a halfspace with Dirichlet boundary condition; cf. [11] and [22].

\subsection{Solution of the quasilinear problem.}

We do not intend to give the detailed proof here, but rather present the main ideas of the proof. In fact, the arguments are very similar to those employed in [12] for the Stefan problem with surface tension, and in [14] for the free boundary value problem of the Navier-Stokes equations.

Introduce function spaces $X_{T} \times Y_{T}$ for the right-hand side of the differential equations in (13) and $Z_{T}$ for the corresponding solutions as follows. The elements of $X_{T}$ are functions

$$
f=\left(f_{v}, f_{w}, f_{p}, f_{c}\right), \quad \text { defined on }[0, T] \times \mathbb{R}^{n+1} \times \dot{\mathbb{R}},
$$

with regularity given by a), c), d) of Theorem 2. The elements of $Y_{T}$ are functions

$$
g=\left(g_{v}, g_{w}, g_{h}, g_{c}, g_{\gamma}\right), \quad \text { defined on }[0, T] \times \mathbb{R}^{n},
$$

with regularity defined in e), f), g), h) of Theorem 2. The solution space $Z_{T}$ consists of functions $z=\left(v, w, p, h, \tilde{c}, \tilde{c}_{\Gamma}\right)$ with regularity as described in Theorem 2. $Z_{T}$ additionally also contains the first of the boundary conditions in (14), i.e. $[v]=0,[w]=0$. Equipped with their natural norms these spaces are Banach spaces. The corresponding spaces of functions with zero trace at $t=0$ (if they exist) will be denoted by a lower left subscript 0 .

Next it is convenient to introduce an auxiliary function $z^{*} \in Z_{T}$ which resolves the initial conditions and the compatibilities j), k) and l) of Theorem 2. By the assumptions of Theorem 1 such a function $z^{*}$ exists, it can be constructed via Theorem 2 applied to special right-hand sides. This way we can now work in the spaces ${ }_{0} Z_{T}$ and ${ }_{0} X_{T} \times{ }_{0} Y_{T}$, which allow for embeddings with embedding constants independent of $T$. The choice of the exponent $p>n+3$ yields

$$
\begin{array}{ll}
\left(v_{ \pm}, w_{ \pm}\right) \in C\left([0, T] ; C_{0}^{1}\left(\mathbb{H}_{ \pm}^{n+1}\right)\right), & \tilde{c} \in C\left([0, T] ; C_{0}^{1}\left(\mathbb{H}_{+}^{n+1}\right)\right), \\
\tilde{c}_{\Gamma} \in C\left([0, T] ; C_{0}^{1}\left(\mathbb{R}^{n+1}\right)\right), & h \in C\left([0, T] ; C_{0}^{2}\left(\mathbb{R}^{n}\right)\right) \cap C^{1}\left([0, T] ; C_{0}^{1}\left(\mathbb{R}^{n}\right)\right),
\end{array}
$$

and the spaces for the $g$ 's are Banach algebras, except for that of $g_{c}$.

Introducing the linear operator $L:{ }_{0} Z_{T} \rightarrow{ }_{0} X_{T} \times{ }_{0} Y_{T}$, defined by the left-hand side of (13) and the last term in (14), Theorem 2 shows that $L$ is linear, bounded, and invertible. The norm of $L^{-1}$ is bounded by some constant $M$, which does not depend on $T \in\left(0, T_{0}\right]$, where $T_{0}<\infty$ is fixed. We now define $H: Z_{T} \rightarrow X_{T} \times Y_{T}$ by the right-hand side of (11) and by the last boundary condition in (12).

Then the nonlinear problem can be rewritten as

$$
L z=H\left(z+z^{*}\right)-L z^{*}:=H_{0}(z), \quad z \in{ }_{0} Z_{T} .
$$

Note that $H_{0}$ maps ${ }_{0} Z_{T}$ into ${ }_{0} X_{T} \times{ }_{0} Y_{T}$ by construction. Inverting $L$, this problem becomes the fixed point problem

$$
z=L^{-1} H_{0}(z), \quad z \in{ }_{0} Z_{T}
$$


Fix a closed ball $\bar{B}_{r}(0) \subset{ }_{0} Z_{T}$. The aim is to show that $L^{-1} H_{0}$ maps $\bar{B}_{r}(0)$ into itself and is a strict contraction on $\bar{B}_{r}(0)$. Thus we have to show that

$$
\left|H_{0}(z)-H_{0}(\bar{z})\right|_{0} X_{T} \times_{0} Y_{T} \leq \frac{1}{2 M}|z-\bar{z}|_{0} Z_{Z}, \quad\left|H_{0}(z)\right|_{0} X_{T} \times_{0} Y_{T} \leq \frac{r}{M}, \quad z, \bar{z} \in \bar{B}_{r}(0) .
$$

Among the finitely many terms appearing in the definition of the $F$ 's and $G$ 's we encounter three types.

- terms containing the factor $\nabla_{x} h$;

- terms containing the factor $\sigma\left(\tilde{c}_{\Gamma}\right)-\sigma_{0}$;

- lower order terms.

Next observe that terms containing derivatives of highest order are linear in these derivatives and are of the first two types. They can be made small by choice of $\eta>0$. On the other hand, lower order terms can be made small by the choice of $T>0$ and $r>0$. These estimates are of the same character as in the two papers cited above.

In conclusion, we can manage this way to show that $L^{-1} H_{0}: \bar{B}_{r}(0) \rightarrow \bar{B}_{r}(0)$ is a strict contraction, and hence admits a unique fixed point, thanks to the contraction mapping principle. This yields a unique solution on a possibly small time interval in $Z_{T}$, the optimal regularity class of type $L_{p}$.

Further regularity of the solutions can be obtained by the parameter trick; cf. [23] and [13]. Under the assumptions of Theorem 1 we obtain in this way classical solutions for $t \in(0, T]$, and even real analyticity in case the given functions $\gamma$ and $\sigma$ have this property. We refrain from giving more details here.

\section{Appendix A. Surface Divergence.}

Let $M$ be an $m$-dimensional $C^{k}$-submanifold of $\mathbb{R}^{n}$. Then, locally around any point $p \in M$, the submanifold is given by a parametrization of class $C^{k}$. Hence there is an open parameter region $B \subset \mathbb{R}^{m}$ and a $C^{k}$-function $g: B \rightarrow \mathbb{R}^{n}$ such that $g(B)=M \cap U$ for some open neighborhood $U \subset \mathbb{R}^{n}$ of $p$. Furthermore, the set

$$
\left\{\partial_{1} g(y), \ldots, \partial_{m} g(y)\right\}
$$

is a linearly independent subset of $\mathbb{R}^{n}$, and $g$ is a homeomorphism from $B$ onto $M \cap U$. The tangent space $T_{p} M$ at $p \in M$ is given by

$$
T_{p} M=\left\{\tau \in \mathbb{R}^{n}: \exists C^{1} \text {-curve } \gamma:(-\epsilon, \epsilon) \rightarrow M \text { s.t. } \gamma(0)=p, \gamma^{\prime}(0)=\tau\right\} .
$$

Then,

$$
T_{p} M=\operatorname{span}\left\{\partial_{1} g(y), \ldots, \partial_{m} g(y)\right\} \quad \text { for } p \in M, y=g^{-1}(p) .
$$

Let $f$ be a vector field on $M$, meaning that $f: M \rightarrow \mathbb{R}^{n}$ is everywhere tangential to $M$, i.e. $f(p) \in T_{p} M$ for all $p \in M$. Such an $f$ is a $C^{1}$-vector field on $M$ if $f \circ g$ is continuously differentiable for every $C^{1}$-parametrization $g$ of $M$. It then follows that $f \circ \gamma$ is continuously differentiable for every $C^{1}$-curve $\gamma:(-\epsilon, \epsilon) \rightarrow M$. In this case, given $p \in M$ and $\tau \in T_{p} M$ with $\tau \neq 0$, the directional derivative of $f$ at $p$ in direction of $\tau$ is

$$
\frac{\partial f}{\partial \tau}(p)=\frac{d}{d t}(f \circ \gamma)(0)
$$

for a $C^{1}$-curve $\gamma:(-\epsilon, \epsilon) \rightarrow M$ such that $\gamma(0)=p, \gamma^{\prime}(0)=\tau$. Correspondingly, a scalar field $\phi: M \rightarrow \mathbb{R}$ is $C^{1}$ if $\phi \circ g$ is $C^{1}$ for every $C^{1}$-parametrization $g$. In this case, the surface gradient $\operatorname{grad}_{M} \phi$ is given by

$$
\operatorname{grad}_{M} \phi(p)=\sum_{i=1}^{m} \frac{\partial \phi}{\partial \tau_{i}} \tau_{i}
$$

where $\left\{\tau_{1}, \ldots, \tau_{m}\right\}$ is an orthonormal basis of $T_{p} M$.

Given a $C^{1}$-vector field $f$ on $M$, a coordinate-free definition of the surface divergence $\operatorname{div}_{M} f$ of $f$ is

$$
\operatorname{div}_{M} f(p)=\lim _{r \rightarrow 0+} \frac{1}{\left|M_{r}(p)\right|} \int_{\partial M_{r}(p)}\langle f, N\rangle d \sigma_{m-1}
$$


where

$$
M_{r}(p)=M \cap V_{r}(p)
$$

with a family of open sets $V_{r}(p) \subset \mathbb{R}^{n}$ such that

$$
p \in V_{r}(p), \operatorname{diam}\left(V_{r}(p)\right)=\mathcal{O}(r) \quad \text { as } r \rightarrow 0+
$$

and $\partial V_{r}(p)$ is piecewise of class $C^{2}$; notice that $M_{r}(p)$ is an $m$-dimensional submanifold with boundary $\partial M_{r}(p)$ and the latter is itself an $(m-1)$-dimensional submanifold of $M$ without boundary. In (16), $\langle\cdot, \cdot\rangle$ is the Euclidean (inner) product on $\mathbb{R}^{n}$ and $N$ denotes the exterior unit normal field to $\partial M_{r}(p)$, i.e. $N(q) \in T_{q} M, N(q) \perp T_{q} \partial M_{r}(p)$ at $q \in \partial M_{r}(p)$ and $N(q)$ points outward w.r. to $M_{r}(p)$. Further, $\left|M_{r}(p)\right|$ denotes the surface area of $M_{r}(p)$. If $M$ has parametrization $g: B \subset \mathbb{R}^{m} \rightarrow \mathbb{R}^{n}$ near $p \in M$, then

$$
\left|M_{r}(p)\right|=\int_{B_{r}} \sqrt{\operatorname{det} G(y)} d y
$$

with $B_{r}=g^{-1}\left(M_{r}(p)\right)$ and the Gramian

$$
G(y)=\left(\left\langle\partial_{i} g(y), \partial_{j} g(y)\right\rangle\right)_{1 \leq i, j \leq m} .
$$

In the following, $d \sigma_{m}=\sqrt{\operatorname{det} G(y)} d y$ denotes the surface measure on $M$ and $d \sigma_{m-1}$ is the corresponding surface measure on the respective $(m-1)$-dimensional boundary-submanifold.

A more common formulation of $\operatorname{div}_{M} f$ is obtained as follows. Given $p \in M$ and a basis $\left\{\tau_{1}, \ldots, \tau_{m}\right\}$ of $T_{p} M$, let $\left\{\tau_{1}^{*}, \ldots, \tau_{m}^{*}\right\} \subset T_{p} M$ denote the co-basis such that

$$
\left\langle\tau_{i}, \tau_{j}^{*}\right\rangle=\delta_{i j}
$$

i.e. $\left\{\tau_{i}\right\},\left\{\tau_{j}^{*}\right\} \subset T_{p} M$ form a biorthogonal system. Extend $\left\{\tau_{1}, \ldots, \tau_{m}\right\}$ by means of $\tau_{m+1}, \ldots, \tau_{n}$ $\in\left(T_{p} M\right)^{\perp}$ to a basis $\left\{\tau_{1}, \ldots, \tau_{n}\right\}$ of $\mathbb{R}^{n}$. We now exploit the fact that $M$ is locally a graph over $T_{p} M$ : Let $\varphi: M \cap B_{\epsilon}(p) \rightarrow T_{p} M$ be given by

$$
\varphi(q)=\sum_{i=1}^{m} y_{i} \tau_{i} \text { for } q \in M \text { with } q=p+\sum_{i=1}^{m} y_{i} \tau_{i}+\sum_{k=m+1}^{n} t_{k} \tau_{k} .
$$

For sufficiently small $\epsilon>0$, the map $\varphi: M \cap B_{\epsilon}(p) \rightarrow \varphi\left(M \cap B_{\epsilon}(p)\right)$ is a diffeomorphism. Now, let $B_{r}=(-r, r)^{m} \subset \mathbb{R}^{m}$ with $r>0$ and define the parametrization $g: B_{r} \rightarrow \mathbb{R}^{n}$ by

Then

$$
g(y)=\varphi^{-1}\left(\sum_{i=1}^{m} y_{i} \tau_{i}\right)
$$

$$
\partial_{i} g(y)=\tau_{i}+o(|y|) \quad \text { as } y \rightarrow 0,
$$

since $\varphi$ approximates the identity near the point $p$. Let

$$
V_{r}(p)=\left\{p+\sum_{i=1}^{n} t_{i} \tau_{i}: t_{i} \in(-r, r)\right\}
$$

and

$$
M_{r}(p)=M \cap V_{r}(p)=g\left(B_{r}\right) \text { for small } r>0
$$

Then

$$
\left|M_{r}(p)\right|=\int_{B_{r}} \sqrt{\operatorname{det}\left(\left\langle\partial_{i} g(y), \partial_{j} g(y)\right\rangle\right)} d y=(2 r)^{m} \sqrt{\operatorname{det} G}+o\left(r^{m}\right) \quad \text { as } r \rightarrow 0+
$$

with $G=\left(\left\langle\tau_{i}, \tau_{j}\right\rangle\right)_{1 \leq i, j \leq m}$. The boundary of $M_{r}(p)$ is

$$
\partial M_{r}(p)=\bigcup_{j=1}^{m} W_{r}^{j}(p) \quad \text { with } \quad W_{r}^{j}(p)=W_{r}^{j,+}(p) \cup W_{r}^{j,-}(p),
$$

where

$$
W_{r}^{j, \pm}(p)=\left\{g(y): y_{i} \in[-r, r] \text { for } i \neq j, y_{j}= \pm r\right\} .
$$

On $\partial M_{r}(p)$ the outer unit normal field $N$ satisfies

$$
N(q)= \pm \frac{\tau_{j}^{*}}{\left|\tau_{j}^{*}\right|}+o(r) \quad \text { for } q \in W_{r}^{j, \pm}(p) .
$$


Hence

$$
\begin{aligned}
& \int_{W_{r}^{j}(p)}\langle f, N\rangle d \sigma_{m-1}=\int_{W_{r}^{j,+}(p)}\langle f, N\rangle d \sigma_{m-1}+\int_{W_{r}^{j,-}(p)}\langle f, N\rangle d \sigma_{m-1} \\
& =\int_{[-r, r]^{m-1}}\left(\left\langle\left.(f \circ g)(y)\right|_{y_{j}=-r} ^{y_{j}=r}, \frac{\tau_{j}^{*}}{\left|\tau_{j}^{*}\right|}\right\rangle+o(r)\right) \sqrt{\operatorname{det} G^{j}(y)} d y^{j} \\
& =\int_{[-r, r]^{m-1}}\left(\int_{-r}^{r}\left\langle\partial_{j}(f \circ g)(0)+o(r), \frac{\tau_{j}^{*}}{\left|\tau_{j}^{*}\right|}\right\rangle d y_{j}+o(r)\right) \sqrt{\operatorname{det} G^{j}(y)} d y^{j} \\
& =(2 r)^{m}\left\langle\frac{\partial f}{\partial \tau_{j}}(p), \frac{\tau_{j}^{*}}{\left|\tau_{j}^{*}\right|}\right\rangle \sqrt{\operatorname{det} G^{j}}+o\left(r^{m}\right) \quad(\text { as } r \rightarrow 0+),
\end{aligned}
$$

where $d y^{j}=d y_{1} \ldots d y_{j-1} d y_{j+1} \ldots d y_{m}$ and the matrix $G^{j}$ is obtained from $G$ by elimination of the $j$-th row and column. Due to the basic geometric fact that "volume = base area $\times$ height", it follows that

$$
\sqrt{\operatorname{det} G}=\sqrt{\operatorname{det} G^{j}}\left\langle\tau_{j}, \frac{\tau_{j}^{*}}{\left|\tau_{j}^{*}\right|}\right\rangle=\frac{1}{\left|\tau_{j}^{*}\right|} \sqrt{\operatorname{det} G^{j}} \quad \text { for } j=1, \ldots, m
$$

Hence,

$$
\operatorname{div}_{M} f(p)=\sum_{j=1}^{m}\left\langle\frac{\partial f}{\partial \tau_{j}}(p), \tau_{j}^{*}\right\rangle
$$

where $\left\{\tau_{i}\right\},\left\{\tau_{j}^{*}\right\} \subset T_{p} M$ is any biorthogonal system. In case $\left\{\tau_{1}, \ldots, \tau_{m}\right\}$ is an orthonormal basis of $T_{p} M,(17)$ resembles the well-known definition of the divergence in $\mathbb{R}^{m}$. For concrete computations it is more useful to write $\operatorname{div}_{M} f(p)$ in the local coordinates that correspond to a given parametrization, i.e. to use

$$
\tau_{j}=\partial_{j} g(y) \quad \text { with } y=g^{-1}(p) .
$$

By definition of $G$, it follows that $\left\{\tau_{j}^{*}\right\}$ is then given by

$$
\left(\tau_{1}^{*}|\ldots| \tau_{m}^{*}\right)=\left(\tau_{1}|\ldots| \tau_{m}\right) G^{-1}
$$

i.e.

$$
\tau_{j}^{*}=\sum_{i=1}^{m} g^{i j} \tau_{i} \quad \text { in case }\left(g^{i j}\right):=G^{-1} .
$$

Inserting this into (17) yields

$$
\operatorname{div}_{M} f(p)=\sum_{i, j=1}^{m} g^{i j}\left\langle\partial_{j}(f \circ g)(y), \partial_{i} g(y)\right\rangle \quad \text { for } y=g^{-1}(p) .
$$

Using the fact that

$$
\operatorname{div}_{M}\left(\frac{1}{\sqrt{\operatorname{det} G}} \tau_{k}\right)=0 \quad \text { for } k=1, \ldots, m
$$

a significant simplification of (19) can be obtained. Indeed,

$$
\operatorname{div}_{M} f=\frac{1}{\sqrt{\operatorname{det} G}} \sum_{j=1}^{m} \partial_{j}\left(\left(v^{j} \circ g\right) \sqrt{\operatorname{det} G}\right)
$$

where the $v^{j}$ denote the components of $f$ w.r. to the basis $\left\{\tau_{j}\right\}$, i.e.

$$
f(p)=\sum_{j=1}^{m} v^{j}(p) \tau_{j}(p) \quad \text { on } M .
$$


Let us briefly justify equation (20). By (17) we have

$$
\begin{aligned}
\operatorname{div}_{M}\left(\frac{1}{\sqrt{\operatorname{det} G}} \tau_{k}\right) & =\sum_{j=1}^{m}\left\langle\partial_{j}\left(\frac{1}{\sqrt{\operatorname{det} G}} \tau_{k}\right), \tau_{j}^{*}\right\rangle \\
& =\frac{1}{\sqrt{\operatorname{det} G}} \sum_{j=1}^{m}\left(-\frac{\partial_{j} \operatorname{det} G}{2 \operatorname{det} G}\left\langle\tau_{k}, \tau_{j}^{*}\right\rangle+\left\langle\partial_{j} \tau_{k}, \tau_{j}^{*}\right\rangle\right) \\
& =\frac{1}{\sqrt{\operatorname{det} G}}\left(-\frac{\partial_{k} \operatorname{det} G}{2 \operatorname{det} G}+\sum_{j=1}^{m}\left\langle\partial_{j} \tau_{k}, \tau_{j}^{*}\right\rangle\right) .
\end{aligned}
$$

The matrix $G=\left(\left\langle\tau_{i}, \tau_{j}\right\rangle\right)$ satisfies the equation $\partial_{k} G=Q G$, with $Q=\left(\partial_{k} G\right) G^{-1}$. According to Liouville's Theorem, the $\operatorname{determinant} \operatorname{det} G$ then satisfies

$$
\partial_{k} \operatorname{det} G=(\operatorname{tr} Q) \operatorname{det} G .
$$

The matrix $Q$ is given by $Q=\left(\partial_{k}\left\langle\tau_{i}, \tau_{j}\right\rangle\right) G^{-1}$. For the trace of $Q$ one obtains

$$
\begin{aligned}
\operatorname{tr} Q & =\sum_{i, j=1}^{m}\left(\left\langle\partial_{k} \tau_{i}, \tau_{j}\right\rangle+\left\langle\tau_{i}, \partial_{k} \tau_{j}\right\rangle\right) g^{i j}=\sum_{i, j=1}^{m}\left(\left\langle\partial_{k} \tau_{i}, g^{i j} \tau_{j}\right\rangle+\left\langle g^{i j} \tau_{i}, \partial_{k} \tau_{j}\right\rangle\right) \\
& =\sum_{i=1}^{m}\left\langle\partial_{k} \tau_{i}, \tau_{i}^{*}\right\rangle+\sum_{j=1}^{m}\left\langle\tau_{j}^{*}, \partial_{k} \tau_{j}\right\rangle=2 \sum_{j=1}^{m}\left\langle\tau_{j}^{*}, \partial_{k} \tau_{j}\right\rangle .
\end{aligned}
$$

Consequently, $\partial_{k} \operatorname{det} G=2 \sum_{j}\left\langle\tau_{j}^{*}, \partial_{k} \tau_{j}\right\rangle \operatorname{det} G$ and (20) follows.

The divergence theorem is inherited from the definition of the surface divergence. It reads as (see Theorem 2.1 in Chapter XII of [20]).

Theorem A (Divergence theorem). Let $M$ be a compact $m$-dimensional $C^{2}$-submanifold of $\mathbb{R}^{n}$ with continuous normal field, and let $N$ be the exterior unit normal field to its boundary $\partial M$. Then, for every $C^{1}$-vector field $f$ on $M$,

$$
\int_{M} \operatorname{div}_{M} f d \sigma_{m}=\int_{\partial M}\langle f, N\rangle d \sigma_{m-1}
$$

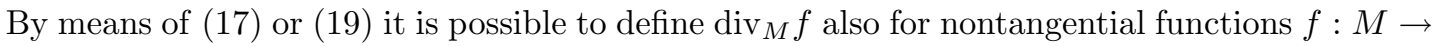
$\mathbb{R}^{n}$. One important example is the surface divergence of the unit normal field of an $(n-1)$ dimensional submanifold (i.e. a hypersurface). In fact, the sum of the principal curvatures of $M$ at $p$ is given by (see for instance p. 223 in [4])

$$
\kappa=-\operatorname{div}_{M} n_{M} \text {. }
$$

Indeed, equation (19) then becomes

$$
-\operatorname{div}_{M} n_{M}=-\sum_{i, j=1}^{m} g^{i j}\left\langle\partial_{j}\left(n_{M} \circ g\right), \partial_{i} g\right\rangle=\sum_{i, j=1}^{m} g^{i j} h_{j i},
$$

where

$$
h_{j i}:=-\left\langle\partial_{j}\left(n_{M} \circ g\right), \partial_{i} g\right\rangle=\left\langle n_{M} \circ g, \partial_{j} \partial_{i} g\right\rangle
$$

is the second fundamental form of $M$ (with respect to the parametrization $g$ ). The expression $\sum_{i, j=1}^{m} g^{i j} h_{j i}$ is the trace of the second fundamental form, and it gives the sum of the principal curvatures of $M$. In case $M$ is the graph of a function $h: B \rightarrow \mathbb{R}$ for some open set $B \subset \mathbb{R}^{m}$, that is, $M:=\{(x, h(x)): x \in B\}$, one easily shows that

and

$$
g^{i j}=\left(\delta_{i j}-\frac{\partial_{i} h \partial_{j} h}{1+|\nabla h|^{2}}\right)
$$

$$
\kappa=\frac{1}{\sqrt{1+|\nabla h|^{2}}} \sum_{i, j=1}^{m}\left(\delta_{i j}-\frac{\partial_{i} h \partial_{j} h}{1+|\nabla h|^{2}}\right) \partial_{i} \partial_{j} h=\operatorname{div}_{\mathbb{R}^{m}}\left(\frac{1}{\sqrt{1+|\nabla h|^{2}}} \nabla h\right)
$$


Let us note in passing that for $n=3$, Gauss called $H=\frac{1}{2} \kappa$ the mean curvature of $M$. Notice also that the divergence theorem does not apply to $f=n_{M}$. For a not necessarily tangent field $f: M \rightarrow \mathbb{R}^{n}$ the decomposition

$$
f=f_{M}+\left\langle f, n_{M}\right\rangle n_{M},
$$

where $f_{M}$ is the tangential part of $f$, leads to the formula

$$
\operatorname{div}_{M} f=\operatorname{div}_{M} f_{M}-\kappa\left\langle f, n_{M}\right\rangle ;
$$

observe that

$$
\operatorname{div}_{M}\left(\left\langle f, n_{M}\right\rangle n_{M}\right)=\left\langle\operatorname{grad}_{M}\left\langle f, n_{M}\right\rangle, n_{M}\right\rangle+\left\langle f, n_{M}\right\rangle \operatorname{div}_{M} n_{M}
$$

and $\operatorname{grad}_{M} \phi(p) \in T_{p} M$ for any scalar field $\phi$. Finally, recall that in local coordinates given by a parametrization $g$, the Laplace-Beltrami operator $\Delta_{M}$, defined by $\Delta_{M} \phi=\operatorname{div}_{M} \operatorname{grad}_{M} \phi$, is given as

$$
\Delta_{M} \phi(p)=\frac{1}{\sqrt{\operatorname{det} G(y)}} \sum_{j, k=1}^{m} \partial_{j}\left(\sqrt{\operatorname{det} G(y)} g^{j k} \partial_{k}(\phi \circ g)(y)\right), \quad y=g^{-1}(p),
$$

for $y \in B$.

For more information concerning vector analysis on (sub-)manifolds see, e.g., [3, 4, 20].

Appendix B. Surface Transport Theorem.

With the notation from $\mathrm{A}$, we are in a position to prove the following result.

Theorem B (Surface transport theorem). Let $I \subset \mathbb{R}$ open, $\Omega \subset \mathbb{R}^{n}$ open and the velocity field $v: J \times \Omega \rightarrow \mathbb{R}^{n}$ be $C^{1}$. Given $t_{0} \in I, x_{0} \in \Omega$ let $\phi\left(t ; t_{0}, x_{0}\right)$ denote the solution of the ODE-system

$$
\dot{\phi}(t)=v(t, \phi(t)), \quad \phi\left(t_{0}\right)=x_{0} .
$$

Given a compact $C^{2}$-hypersurface $M_{0} \subset \Omega$, let

$$
M_{t}=\left\{\phi\left(t ; t_{0}, x_{0}\right): x_{0} \in M_{0}\right\}
$$

denote the advected (material) surface; due to the assumptions on $v, \Omega$ and $M_{0}$ the surface $M_{t}$ exists for all $t \in I_{\delta}=\left(t_{0}-\delta, t_{0}+\delta\right)$ for some $\delta>0$. Let

$$
c: \operatorname{gr}\left(M_{t}\right)=\left\{(t, x): t \in I_{\delta}, x \in M_{t}\right\} \rightarrow \mathbb{R}
$$

be continuously differentiable. Then,

$$
\begin{aligned}
\frac{d}{d t} \int_{M_{t}} c d \sigma_{t} & =\int_{M_{t}}\left(\frac{D c}{D t}+c \operatorname{div}_{M} v\right) d \sigma_{t} \\
& =\int_{M_{t}}\left(\frac{D c}{D t}+c \operatorname{div}_{M} v_{M}-c \kappa V\right) d \sigma_{t}
\end{aligned}
$$

for all $t \in I_{\delta}$. Here, d $\sigma_{t}$ denotes the surface measure on $M_{t}, \kappa=\kappa(t, x)$ the curvature of $\Gamma(t)$ at $x \in \Gamma(t)$ according to (23), $v_{M}$ the tangential part of $v, V=\left\langle v, n_{M}\right\rangle$ the normal component of $v$, and

$$
\frac{D c}{D t}(t, x)=\frac{d}{d s} c(t+s, \phi(t+s ; t, x))_{\mid s=0}
$$

is the Lagrangian derivative of $g$.

Proof. Let $m=n-1, g: B \rightarrow \mathbb{R}^{n}$, with $B \subset \mathbb{R}^{m}$ open and bounded, be a parametrization of $M_{0}$ and $\Phi^{t}(y)=\phi\left(t ; t_{0}, y\right)$ be the flow map associated with $v$. Then,

$$
g^{t}: B \rightarrow \mathbb{R}^{n} \quad \text { with } g^{t}:=\Phi^{t} \circ g
$$

is a parametrization of $M_{t}$. Hence,

$$
\int_{M_{t}} c(t, x) d \sigma_{t}=\int_{B} c\left(t, \Phi^{t}(y)\right) \sqrt{\operatorname{det} G(t, y)} d y,
$$


where $G(t, y)$ is the Gramian

$$
G(t, y)=\left(\left\langle\partial_{i} g^{t}(y), \partial_{j} g^{t}(y)\right\rangle\right)_{1 \leq i, j \leq m} .
$$

Therefore,

$$
\begin{aligned}
& \frac{d}{d t} \int_{M_{t}} c(t, x) d \sigma_{t} \\
& \quad=\int_{B}\left(\frac{d}{d t} c\left(t, \Phi^{t}(y)\right)\right) \sqrt{\operatorname{det} G(t, y)} d y+\int_{B} \frac{c\left(t, \Phi^{t}(y)\right)}{\sqrt{\operatorname{det} G(t, y)}} \frac{1}{2} \partial_{t} \operatorname{det} G(t, y) d y
\end{aligned}
$$

and, by the definition of $\frac{D c}{D t}$, the first term on the right is

$$
\int_{B}\left(\frac{d}{d t} c\left(t, \Phi^{t}(y)\right)\right) \sqrt{\operatorname{det} G(t, y)} d y=\int_{M_{t}} \frac{D c}{D t}(t, x) d \sigma_{t} .
$$

The derivative of $\partial_{t} \operatorname{det} G(t, y)$ can be computed by using Liouville's Theorem. However, we prefer here to give a self-contained derivation. For this purpose, notice first that

$$
\partial_{t} \operatorname{det} G(t, y)=\sum_{j=1}^{m} \operatorname{det}\left(\begin{array}{ccccc}
\left\langle\partial_{1} g^{t}, \partial_{1} g^{t}\right\rangle & \cdots & \partial_{t}\left\langle\partial_{1} g^{t}, \partial_{j} g^{t}\right\rangle & \cdots & \left\langle\partial_{1} g^{t}, \partial_{m} g^{t}\right\rangle \\
\vdots & & \vdots & & \vdots \\
\left\langle\partial_{m} g^{t}, \partial_{1} g^{t}\right\rangle & \cdots & \partial_{t}\left\langle\partial_{m} g^{t}, \partial_{j} g^{t}\right\rangle & \cdots & \left\langle\partial_{m} g^{t}, \partial_{m} g^{t}\right\rangle
\end{array}\right) .
$$

Using $\partial_{t} \partial_{j} g^{t}(y)=\nabla_{x} v\left(t, g^{t}(y)\right) \partial_{j} g^{t}(y)$ we obtain

$$
\frac{1}{2} \partial_{t}\left\langle\partial_{i} g^{t}(y), \partial_{j} g^{t}(y)\right\rangle=\left\langle D \partial_{i} g^{t}(y), \partial_{j} g^{t}(y)\right\rangle
$$

with

$$
D=\frac{1}{2}\left(\nabla_{x} v+\left(\nabla_{x} v\right)^{T}\right), \quad\left(\nabla_{x} v\right)^{T}=\left(\nabla_{x} v_{1}|\ldots| \nabla_{x} v_{n}\right) .
$$

Consequently, if $\tau_{i}=\partial_{i} g^{t}$, then

$$
\frac{1}{2} \partial_{t} \operatorname{det} G(t, y)=\sum_{j=1}^{m} \operatorname{det}\left(\begin{array}{ccccc}
\left\langle\tau_{1}, \tau_{1}\right\rangle & \cdots & \left\langle D \tau_{1}, \tau_{j}\right\rangle & \cdots & \left\langle\tau_{1}, \tau_{m}\right\rangle \\
\vdots & & \vdots & & \vdots \\
\left\langle\tau_{m}, \tau_{1}\right\rangle & \cdots & \left\langle D \tau_{m}, \tau_{j}\right\rangle & \cdots & \left\langle\tau_{m}, \tau_{m}\right\rangle
\end{array}\right) .
$$

Define $Q \in \mathbb{R}^{m \times m}$ by means of

$$
Q G=Q\left(\left\langle\tau_{i}, \tau_{j}\right\rangle\right)=\left(\left\langle D \tau_{i}, \tau_{j}\right\rangle\right) .
$$

Let $a_{i j}=\left\langle\tau_{i}, \tau_{j}\right\rangle$ and $\alpha^{i}=\left(\alpha_{i 1}, \ldots, \alpha_{i m}\right)^{T}$. Then

$$
\frac{1}{2} \partial_{t} \operatorname{det} G(t, y)=\sum_{j=1}^{m} \operatorname{det}\left(\alpha^{1}|\ldots| Q \alpha^{j}|\ldots| \alpha^{m}\right) .
$$

Due to the properties of the determinant it is now easy to obtain

$$
\frac{1}{2} \partial_{t} \operatorname{det} G(t, y)=\operatorname{tr} Q \operatorname{det}\left(\alpha^{1}|\ldots| \alpha^{m}\right)=\operatorname{tr} Q \operatorname{det} G(t, y) .
$$

By (28), the matrix $Q$ satisfies $Q=\left(\left\langle D \tau_{i}, \tau_{j}\right\rangle\right) G(t, y)^{-1}$, hence

$$
Q_{i j}=\sum_{k=1}^{m}\left\langle D \tau_{i}, \tau_{k}\right\rangle g^{k j} \quad \text { if }\left(g^{i j}\right):=G^{-1} .
$$

This yields

$$
Q_{i j}=\left\langle D \tau_{i}, \sum_{k=1}^{m} g^{k j} \tau_{k}\right\rangle=\left\langle D \tau_{i}, \tau_{j}^{*}\right\rangle
$$

with $\tau_{j}^{*}$ according to (18), i.e. $\left\{\tau_{1}^{*}, \ldots, \tau_{m}^{*}\right\} \subset \operatorname{span}\left\{\tau_{1}, \ldots, \tau_{m}\right\}$ is a co-basis. Therefore

$$
\operatorname{tr} Q=\sum_{i=1}^{m} \frac{1}{2}\left(\left\langle\left(\nabla_{x} v\right) \tau_{i}, \tau_{i}^{*}\right\rangle+\left\langle\left(\nabla_{x} v\right) \tau_{i}^{*}, \tau_{i}\right\rangle\right),
$$


hence

$$
\frac{1}{2} \partial_{t} \operatorname{det} G(t, y)=\operatorname{div}_{M_{t}} v\left(t, g^{t}(y)\right) \operatorname{det} G(t, y)
$$

by (17). Insertion into (27) finally yields

$$
\begin{aligned}
& \frac{d}{d t} \int_{M_{t}} c(t, x) d \sigma_{t} \\
& \quad=\int_{B}\left(\frac{d}{d t} c\left(t, \Phi^{t}(y)\right)\right) \sqrt{\operatorname{det} G(t, y)} d y+\int_{B} c\left(t, \Phi^{t}(y)\right) \operatorname{div}_{M_{t}} v\left(t, g^{t}(y)\right) \sqrt{\operatorname{det} G(t, y)} d y \\
& =\int_{M_{t}}\left(\frac{D c}{D t}(t, x)+c(t, x) \operatorname{div}_{M_{t}} v(t, x)\right) d \sigma_{t} .
\end{aligned}
$$

The literature contains several variants of Theorem B (cf. [35]), sometimes with uncommon notations. One such variant of $(26)$ is

$$
\frac{d}{d t} \int_{M_{t}} c d \sigma_{t}=\int_{M_{t}}\left(\left[\frac{\partial c}{\partial t}\right]_{n}+\operatorname{div}_{M}\left(c v_{M}\right)-c \kappa V\right) d \sigma_{t}
$$

where

$$
\left[\frac{\partial c}{\partial t}\right]_{n}(t, x)=\frac{d}{d s} c\left(t+s, \phi_{n}(t+s ; t, x)\right)_{\mid s=0}
$$

with $\phi_{n}$ the purely normal interface motion. In the sections above, we used the notation $\partial_{t, n} c$ instead of $\left[\frac{\partial c}{\partial t}\right]_{n}$ to avoid confusion with the interfacial jump of a quantity. The function $\phi_{n}$ is the solution of

$$
\dot{\phi}_{n}(t)=v_{n}\left(t, \phi_{n}(t)\right), \quad \phi_{n}\left(t_{0}\right)=x_{0},
$$

where

$$
v_{n}(t, x)=\left\langle v(t, x), n_{M}(x)\right\rangle n_{M}(x) \quad \text { on } \operatorname{gr}\left(M_{t}\right) .
$$

The existence of a solution $\phi_{n}$ of (30) is not obvious, since $v_{n}(t, x)$ is only defined on $\operatorname{gr}\left(M_{t}\right)$. In such a situation, the tangency condition

$$
\lim _{h \rightarrow 0+} \frac{1}{h} \operatorname{dist}\left(x_{0}+h v_{n}\left(t_{0}, x_{0}\right), M_{t_{0}+h}\right)=0 \quad \text { for all } t_{0} \text { and } x_{0} \in M_{t_{0}}
$$

is necessary and sufficient for local existence of solutions, given that $M_{t}$ enjoys some mild regularity which holds if $\operatorname{gr}\left(M_{t}\right)$ is a $C^{1}$-submanifold of $\mathbb{R}^{n+1}$ and $v_{n}$ is locally Lipschitz continuous; see, e.g., [6]. Now, given $x_{0} \in M_{t_{0}}$, the $C^{1}$-regularity of $M_{t_{0}}$ and the fact that $v-v_{n}$ with $v=v\left(t_{0}, x_{0}\right)$ and $v_{n}=v_{n}\left(t_{0}, x_{0}\right)$ is tangent to $M_{t_{0}}$ at $x_{0}$ yields

$$
x_{0}-h\left(v-v_{n}\right)+e_{h} \in M_{t_{0}} \quad \text { for small } h>0
$$

with certain $e_{h} \in \mathbb{R}^{n}$ such that $\left|e_{h}\right|=o(h)$ as $h \rightarrow 0+$. This implies

$$
\begin{aligned}
\operatorname{dist}\left(x_{0}+h v_{n}, M_{t_{0}+h}\right) & \leq\left|x_{0}+h v_{n}-\phi\left(t_{0}+h ; t_{0}, x_{0}-h\left(v-v_{n}\right)+e_{h}\right)\right| \\
& =\left|x_{0}+h v_{n}-\left(x_{0}-h\left(v-v_{n}\right)+h v\left(t_{0}, x_{0}-h\left(v-v_{n}\right)\right)\right)\right|+o(h) \\
& =h\left|v\left(t_{0}, x_{0}\right)-v\left(t_{0}, x_{0}-h\left(v-v_{n}\right)\right)\right|+o(h)=o(h) \quad \text { as } h \rightarrow 0+
\end{aligned}
$$

if $v$ is continuous. Hence the required tangency condition holds.

Application of Theorem A yields the formulation

$$
\frac{d}{d t} \int_{M_{t}} c d \sigma_{t}=\int_{M_{t}}\left(\left[\frac{\partial c}{\partial t}\right]_{n}-c \kappa V\right) d \sigma_{t}+\int_{\partial M_{t}} c\langle v, N\rangle d s_{t},
$$

where $d s_{t}$ denotes the surface measure on $\partial M_{t}$. This form of the surface transport theorem nicely displays the different contributions to the change in surfactant mass due to transport of species and geometrical changes. A more formal derivation of Theorem B in the language of Differential Geometry can be found in [4], p. 228ff, while a more heuristic version for $n=3$ is given in [35]. For purely tangential surface motion Theorem B reduces to Theorem 2.11 in Chapter XII of [3], while for purely normal surface motion a generalization to $M_{t} \cap V$ with fixed $V \subset \mathbb{R}^{n}$ instead of $M_{t}$ is given in [15]; for extensions cf. Remark 3 in [15] and [17, 21]. 


\section{References}

[1] F. Abergel, C. Dupaix: Existence of smooth, stationary interfaces for Marangoni-type flow. Nonl. Anal., Theory Meth. Appl. 27(11), 1329-1350 (1996).

[2] G. Allain: Small-time existence for the Navier-Stokes equations with a free surface. Appl. Math. Optim. 16, 37-50 (1987).

[3] H. Amann, J. Escher: Analysis III. Birkhäuser, 2001.

[4] R. Aris: Vectors, tensors, and the basic equations of fluid mechanics. Dover Publications, 1989.

[5] J.T. Beale: Large-time regularity of viscous surface waves. Arch. Rational Mech. Anal. 84, 307-352 (1984).

[6] D. Bothe: Multivalued differential equations on graphs. Nonl. Anal., Theory Meth. Appl. 18(3), 245-252 (1992).

[7] R. Clift, J.R. Grace, M.E. Weber: Bubbles, Drops, and Particles. Academic Press, New York, 1978.

[8] R. Defay, I. Prigogine, A. Bellemans: Surface tension and adsorption. Wiley and Sons, New York, 1966.

[9] I.V. Denisova: Evolution of compressible and incompressible fluids separated by a closed interface. Interfaces Free Bound. 2(3), 283-312 (2000).

[10] I.V. Denisova, V.A. Solonnikov: Classical solvability of the problem on the motion of two viscous incompressible fluids. St. Petersburg Math. J. 7(5), 755-786 (1996); translation from Algebra Anal. 7(5), 101-142 (1995).

[11] R. Denk, M. Hieber, J. Prüss: R-boundedness, Fourier multipliers and problems of elliptic and parabolic type. Mem. Amer. Math. Soc. 166(788), 2003.

[12] J. Escher, J. Prüss, G. Simonett: Analytic solutions for a Stefan problem with Gibbs-Thomson correction. J. reine angew. Math. 563, 1-52 (2003).

[13] J. Escher, J. Prüss, G. Simonett: A new approach to the regularity of solutions for parabolic equations. In: Evolution Equations. Lecture Notes in Pure and Appl. Math. 234, 167-190. Dekker, New York, 2003.

[14] J. Escher, J. Prüss, G. Simonett: Analytic solutions of the free boundary value problem for the two-phase Navier-Stokes system. Preprint.

[15] M.E. Gurtin, A. Struthers, W.O. Williams: A transport theorem for moving interfaces. Quarterly of Applied Mathematics 47(4), 773-777 (1989).

[16] M. Ishii: Thermo-Fluid Dynamic Theory of Two-Phase Flow. Eyrolles, Paris 1975.

[17] J.P. Jaric: On a transport theorem for moving interface. Int. J. Engng. Sci. 30(10), 1535-1542 (1992).

[18] A.J. James, J. Lowengrub: A surfactant-conserving volume-of-fluid method for interfacial flows with insoluble surfactant. J. Comp. Phys. 201(2), 685-722 (2004).

[19] M.V. Lagunova: On the solvability of a three-dimensional problem of thermocapillary convection. J. Sov. Math. 64(6), 1233-1240 (1993); translation from Probl. Math. Anal. 11, 18-27 (1990).

[20] S. Lang: Differential and Riemannian Manifolds. Springer, New York 1995.

[21] H. Petryk, Z. Mroz: Time derivates of integrals and functionals defined on varying volume and surface domains. Arch. Mech. 38(5-6), 697-724 (1986).

[22] J. Prüss: Maximal regularity for abstract parabolic problems with inhomogeneous boundary data in $L_{p}$-spaces. Math. Bohem. 127(2), 311-327 (2002).

[23] J. Prüss: Maximal regularity for evolution equations in $L_{p}$-spaces. Conf. Semin. Mat. Univ. Bari (2002) 285, 1-39 (2003).

[24] F. Ravera, M. Ferrari, L. Liggieri: Adsorption and partitioning of surfactants in liquid-liquid systems. Adv. Coll. Int. Sci. 88, 129-177 (2000).

[25] M. Siegel: Influence of surfactant on rounded and pointed bubbles in two-dimensional Stokes flow. SIAM J. Appl. Math. 59(6), 1998-2027 (1999).

[26] J.C. Slattery: Advanced Transport Phenomena. Cambridge University Press, Cambridge 1999.

[27] V.A. Solonnikov: On the quasistationary approximation in the problem of motion of a capillary drop. pp. 643-671 in Topics in Nonlinear Analysis. The Hermann Amann Anniversary Volume, (J. Escher, G. Simonett, eds.). Birkhäuser, Basel, 1999.

[28] N. Tanaka: Global existence of two-phase non-homogeneous viscous incompressible fluid. Commun. Partial Differ. Equations 18(1-2), 41-81 (1993).

[29] N. Tanaka: Two-phase free boundary problem for viscous incompressible thermo-capillary convection. Jap. J. Math., New Ser. 21(1), 1-42 (1995).

[30] A. Tani: Two-phase free boundary problem for compressible viscous fluid motion. J. Math. Kyoto Univ. 24, 243-267 (1984). 
[31] A. Tani: Small-time existence for the three-dimensional Navier-Stokes equations for an incompressible fluid with a free surface. Arch. Rat. Mech. Anal. 133, 299-331 (1996).

[32] A. Tani, N. Tanaka: Large-time existence of surface waves in incompressible viscous fluids with or without surface tension. Arch. Rat. Mech. Anal. 130, 303-314 (1995).

[33] Y. Teramato: On the Navier-Stokes flow down an inclined plane. J. Math. Kyoto Univ. 32, 593-619 (1992).

[34] A. Wagner: Nonstationary Marangoni convection. Appl. Math. 26(2), 195-220 (1999).

[35] H. Wong, D. Rumschitzki, C. Maldarelli: On the surfactant mass balance at a deforming fluid interface. Phys. Fluids 8(11), 3203-3204 (1996).

Institut für Mathematik \& Department Chemie, Technische Chemie und Chemische Verfahrenstechnik, Universität Paderborn, Warburger Str. 100, D-33098 Paderborn, GERMANY

E-mail address: bothe@tc.uni-paderborn.de

Fachbereich Mathematik und Informatik, Martin-Luther-Universität Halle-WittenBerg, D-60120 Halle, Germany

E-mail address: pruess@mathematik.uni-halle.de

Department of Mathematics, Vanderbilt University, Nashville, TN 37240, USA

E-mail address: simonett@math.vanderbilt.edu 